\title{
Infrared phononic nanoantennas: Localized surface phonon polaritons in $\mathrm{SiC}$ disks
}

\author{
AMEEN Mohamed ${ }^{1}$, GARCIA-ETXARRI Aitzol ${ }^{2}$, SCHNELL Martin $^{3}$, \\ HILLENBRAND Rainer ${ }^{3,4} \&$ AIZPURUA Javier ${ }^{2 *}$ \\ ${ }^{1}$ Department of Materials Physics, University of the Basque Country UPV/EHU, Donostia-San Sebastián 20018, Spain; \\ ${ }^{2}$ Centro Mixto de Física de Materiales CSIC-UPV/EHU and Donostia International Physics Centre, San Sebastian 20018, Spain; \\ ${ }^{3}$ Nanooptics Group, CIC Nanogune Consolider, San Sebastian 20018, Spain; \\ ${ }^{4}$ Ikerbasque, Bilbao 48011, Spain
}

Received April 7, 2010; accepted May 16, 2010

\begin{abstract}
The electromagnetic interaction of light with polar materials shows a sharp and well defined electromagnetic response in the infrared (IR) region that consists mainly of excitation of optical phonons. Similar to surface plasmons in the visible region, surface phonons can couple efficiently to infrared light in micron-sized antennas made of polar materials. We applied the boundary element method to calculating the infrared electromagnetic response of single SiC disks acting as effective infrared antennas as a function of different parameters such as disk size and thickness. We also analyzed the effect of locating a probing metallic tip near the $\mathrm{SiC}$ disk to scatter light in the proximity of the $\mathrm{SiC}$ disk, thereby obtaining new spectral peaks connected with localized modes between the tip and the SiC disk. We then further investigated their application in IR scanning probe microscopy. A near-field map of the phononic resonances enhances the understanding of the nature of the IR extinction peaks.
\end{abstract}

surface phonon polaritons, infrared nanoantennas, polar materials, scattering calculations, scanning IR near-field microscopy

Citation: Ameen M, Garcia-Etxarri A, Schnell M, et al. Infrared phononic nanoantennas: Localized surface phonon polaritons in SiC disks. Chinese Sci Bull, 2010, 55: 2625-2628, doi: 10.1007/s11434-010-4032-4

Nanoantennas are nanoscale structures that effectively receive, transmit and/or emit electromagnetic radiation at certain particular wavelengths that correspond to the electromagnetic resonances of the nanostructure. In the visible region, one of the most effective resonances supporting antenna effects is given by the collective-oscillation modes of the surface charge density at a metallic/dielectric interface, the so-called surface plasmons. The energy (wavelength) of the surface plasmons falls in the optical range of the visible spectrum and the exact spectral position of the resonance depends on the electron density of the metal, the size, the shape and the dielectric surrounding of the nanoantenna, and on the interactions with neighboring structures [1-5].

*Corresponding author (email: aizpurua@ehu.es)
The dielectric contrast between a metal and a dielectric is responsible for the localization of the surface charge density at the surface since the dielectric response of a standard metal has negative values for visible frequencies below the plasma frequency of the metal. Typical metals for optical nanoantenna synthesis are silver and gold. Surface phonon polaritons are the counter partner of surface plasmons but in the infrared (IR) range of the spectrum instead of the visible region. At IR wavelengths, polar materials also show negative values of the dielectric function for frequencies between the transverse and longitudinal optical phonons of the material [6]. The negative/positive dielectric contrast at the polar material/dielectric interface is responsible for localizing the oscillations of the lattice at the interface between the polar material and the dielectric, giving rise to similar pho- 
non-polariton propagation [7] and antenna effects [8-10], where the electromagnetic resonances fall in the IR region. Similar to surface plasmon polaritons in the visible region, the IR surface phonon polariton resonances will depend on the properties of the polar material, the size, the shape and the interactions with the surrounding medium and other scattering objects [11]. We explore here the response of a $\mathrm{SiC}$ disk, as an example of a phononic IR antenna. SiC presents a very clear and narrow response in the IR region [6], which makes it a very suitable material for investigating effective antenna response. This type of antenna can have a major impact in surface-enhanced IR spectroscopies such as in surface-enhanced infrared absorption-reflectance [12] where the vibrational signal of the molecular groups is directly detectable in the transmission mode thanks to the antenna effect in the IR region being complementary to the field-enhancement necessary in the visible region for surface enhanced Raman spectroscopy [13]. The use of a phononic antenna can optimize the enhancing effect of the spectroscopic signal [14], thereby lowering the detection limit of the amounts of molecular groups [12]. Another aspect of the antenna response in the IR region is related to the phase interaction between the molecular groups and the antenna response that can lead to a dramatic improvement in the contrast and lineshape modification due to the interference of the antenna and the sample response [15]. To develop all of these aspects further, it is necessary to know the basic principles of the single phononic antenna response in the IR region, which is reported here in this paper.

We performed full electrodynamical calculations of the IR response of single $\mathrm{SiC}$ disks of different sizes (diameter between $400 \mathrm{~nm}$ and $600 \mathrm{~nm}$, and thickness between $50 \mathrm{~nm}$ and $100 \mathrm{~nm}$ ) using the boundary element method (BEM) to solve Maxwell's equation [16]. The extinction cross-section of a single $\mathrm{SiC}$ disk at the nanoscale shows a typical dipolar peak which consists of an excitation of a disk dipole with positive and negative charges at either side of the disk. This dipolar mode is what we describe as the phononic antenna resonance. A clear dependence of the peak resonance on the size of the disk can be observed in Figure 1(a) for a disk with a fixed thickness of $50 \mathrm{~nm}$. The peak of the extinction spectrum is red shifted when the diameter of the disk is increased due to a larger polarizability of the structure. Because the absorption and scattering cross-sections of an object is proportional to the volume and the square of the volume, respectively, a larger sized disk increases the strength of the extinction and red shifts the response due to the smaller energy of the phononic oscillator.

The dependence of the antenna response on the thickness of the SiC disk, on the other hand, is governed by a different aspect of the antenna resonance, namely, the surface mode interaction. In this case, when the thickness of the disk is reduced, the Coulomb interaction between the top and bottom surfaces of the nanodisk is increased and a new coupled resonance is generated corresponding to a symmetric solu- tion of the surface modes; the so-called bonding mode is analogous to the symmetric solutions of molecular orbitals in quantum chemistry [17]. This effect is observed in Figure 1(b) where the extinction spectrum of a $\mathrm{SiC}$ disk of a radius $500 \mathrm{~nm}$ is represented for disks of different thicknesses. As the thickness of the disk is reduced from $100 \mathrm{~nm}$ down to 50 $\mathrm{nm}$, the dipolar response peak red shifts, following the natural tendency of the symmetric surface phonon solution when the interaction between the top and bottom interfaces is increased.

Interestingly, antenna response for application in surface-enhanced spectroscopies is connected with the distribution of the near-field region. In Figure 1(c) and (d), we show two different cross-sections of the near-field amplitude distribution $|E|$ in the proximity of the $\mathrm{SiC}$ nanodisk (top and side cross-sections) for an incident planewave of wavelength $\lambda=12.106 \mu \mathrm{m}$, which corresponds to the dipolar resonance of a $\mathrm{SiC}$ disk of $500 \mathrm{~nm}$ radius and $50 \mathrm{~nm}$ thickness (blue line in Figure 1(a) and (b)). The dipolar distribution of the local field can be clearly observed both in the top and side views of the near-field region. This field distribution determines the areas of the nanoantenna where the signal from the molecular groups is more efficiently enhanced. Therefore, the ability to access information on large fieldenhancement areas is crucial for effective nanoantenna design.

Optical and infrared antennas are commonly probed using scattering tips to access the near-field distribution around the antennas. To study how the presence of a metallic tip, commonly used in scattering-type near-field optical microscopy [18], can affect the spectral properties of phononic tip scattering, we performed scattering calculations in a situation where the same $\mathrm{SiC}$ disk analyzed previously is probed by a metallic platinum $(\mathrm{Pt})$ tip located in the central position of the $\mathrm{SiC}$ disk. To simulate this situation, we chose a Pt tip with a height of $1 \mu \mathrm{m}$ and a tip-apex radius of $30 \mathrm{~nm}$ under an oblique side-illumination with a p-polarized planewave. As observed in Figure 2, the presence of the $\mathrm{Pt}$ tip results in the appearance of new extinction peaks, which are a consequence of the interaction between the metallic tip and phononic antenna. In particular, in addition to the standard dipolar peak of the single SiC disk also present in the presence of the tip $(\lambda \approx 12.10 \mu \mathrm{m})$, a relatively large new peak appears at a shorter wavelength $(\lambda \approx 11.6 \mu \mathrm{m})$, although it is a weaker signal. These new peaks can be obviously associated to the interaction between the tip and antenna sample.

Because of the near-field region at the apex, the resonant interaction between this localized field at the tip and $\mathrm{SiC}$ excites locally surface phonon polaritons. This effect is therefore mainly associated with the mirror image of the field at the tip apex, which is expected to be independent of the tip shape. While the dipolar resonance is a resonance determined by the global characteristics of the disk, this 

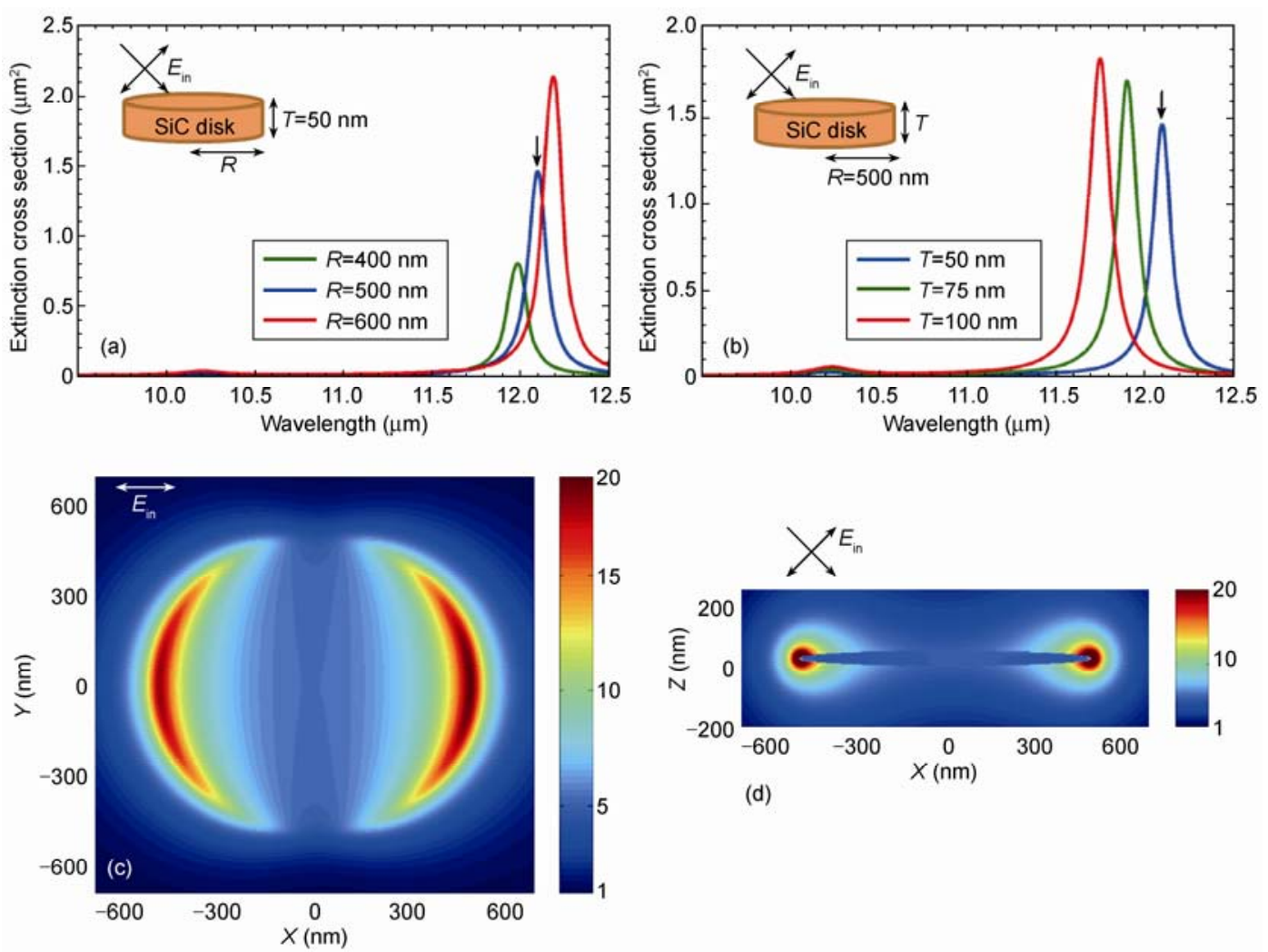

(d)

Figure 1 (a) Extinction spectra for a SiC disk of $50 \mathrm{~nm}$ thickness $T$ and radius $R=400 \mathrm{~nm}$ (green line), $500 \mathrm{~nm}$ (blue line) and $600 \mathrm{~nm}$ (red line); (b) extinction spectra for a SiC disk of $500 \mathrm{~nm}$ radius and thickness $T=50 \mathrm{~nm}$, (blue line), $T=75 \mathrm{~nm}$ (green line) and $T=100 \mathrm{~nm}$ (red line); (c) top view of the near-field amplitude distribution $|E|$ on top of a SiC disk with thickness $T=50 \mathrm{~nm}$ and radius=500 $\mathrm{nm}$ at a wavelength $\lambda=12.106 \mu \mathrm{m}$ which coincides with the dipolar resonances in (a) and (b), marked with an arrow; (d) side view of the same situation as in (c).

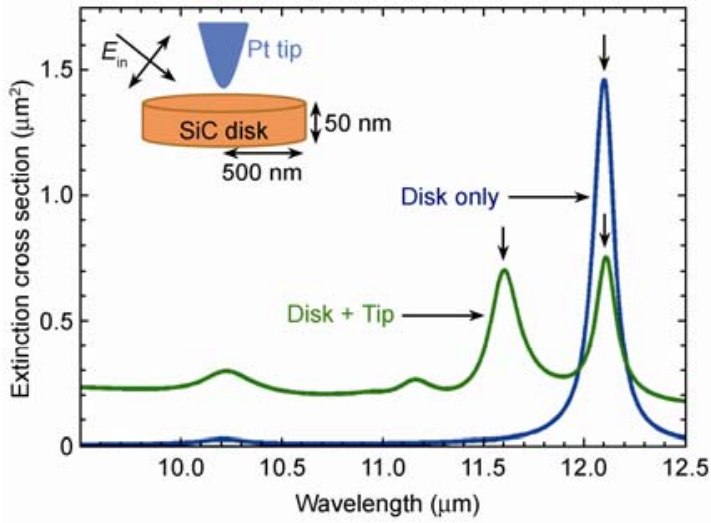

Figure 2 Extinction spectra of a SiC disk of $50 \mathrm{~nm}$ thickness and $500 \mathrm{~nm}$ radius for a planewave at an oblique incidence in a situation where the disk is isolated (blue line), and in a situation where the disk is in close proximity (distance $=6 \mathrm{~nm})$ to a metallic Pt tip (green line).

new peak is due to a local effect.

To obtain insights about the nature of the new extinction peaks, we calculated the near-field amplitude distribution $|E|$ corresponding to the resonance wavelengths of both peaks $(\lambda=12.106 \mu \mathrm{m}$ and $\lambda=11.602 \mu \mathrm{m})$. The results are displayed in Figure 3. At a wavelength of $\lambda=12.106 \mu \mathrm{m}$, i.e. the stronger coupled mode, a near-field distribution is generated both at the edges of the SiC antenna, mimicking a dipolar mode (see Figure 3(a) and (b)) together with a stronger localization of the near-field region directly beneath the Pt tip at the tip-antenna cavity. This mode is therefore a coupled mode that combines the dipolar aspects of the full $\mathrm{SiC}$ antenna together with the localization of the field due to the mirror image of the tip by the $\mathrm{SiC}$ surface. At a wavelength of $\lambda=11.602 \mu \mathrm{m}$ (weaker extinction peak), the near-field region is localized exclusively at the SiC disk-tip cavity (see Figure 3(c) and (d)), meaning that this mode is less radiative.

An extremely interesting aspect of the field distribution of the mode at $\lambda=11.602 \mu \mathrm{m}$ can be observed in Figure 3(c), where the field at the cavity is imaged on the other side of the SiC disk (see field localization below the bottom interface of the disk). This presents clear evidence of the superlensing effect [19], which is possible for this mode because the dielectric response of the $\mathrm{SiC}$ material presents a value close to -1 at this particular wavelength with very few losses (small imaginary part of the dielectric function). The effect of the superlensing allows for faithful imaging of a nanosized object without the restrictions imposed by the diffraction limit that has been proposed theoretically by Pendry [20] and experimentally confirmed both in the IR region with the use of polar materials [19] and in the visible region with the use of a metallic slab [21]. In both cases, the 

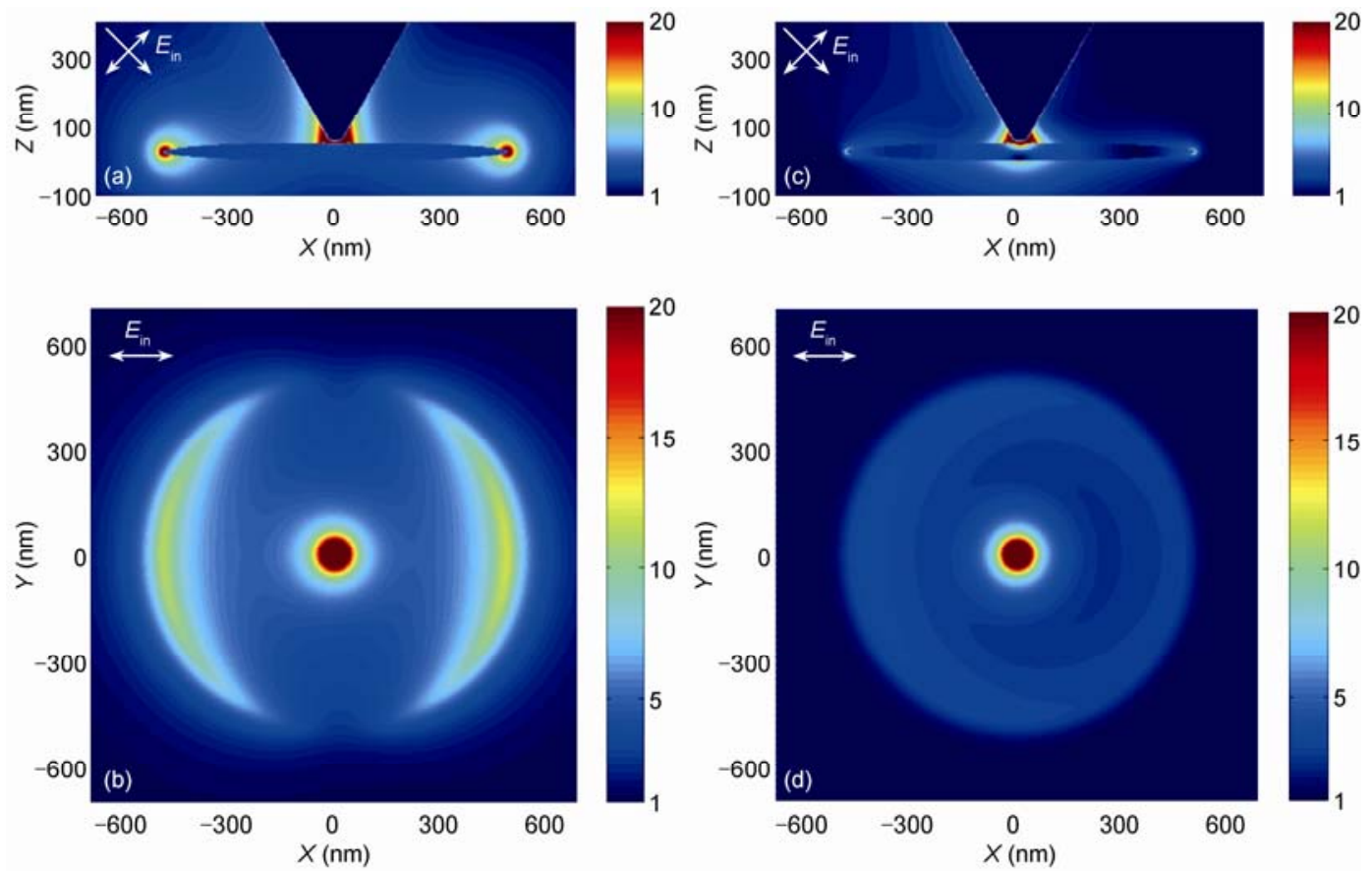

Figure 3 (a) Side view of the near-field amplitude distribution $|E|$ in a SiC disk in the presence of a Pt tip at a separation distance of $6 \mathrm{~nm}$ at a wavelength of the incoming planewave of $12.106 \mu \mathrm{m}$; (b) top view of the same situation as in (a); (c) side view of the amplitude distribution of the near-field region in the same situation as in (a) and (b) at a wavelength of $11.60 \mu \mathrm{m}$; (d) top view of the same situation as in (c).

superlensing effect is restricted to a fixed wavelength, as found here. This situation opens the door for phononic materials of adequate shape (slabs) to behave as perfect IR lenses in addition to their potential for field-enhancing capacities in spectroscopy.

1 Mühlschlegel P, Eisler H J, Martin O J F, et al. Science, 2005, 308: $1607-1609$

2 Fromm D P, Sundaramurthy A, Schuck P J, et al. Nano Lett, 2004, 4: 957-961

3 Taminiau T H, Stefani F D, Segerink F B, et al. Nat Photon, 2008, 2: 234-237

4 Pelton M, Aizpurua J, Bryant G B. Laser Photon Rev, 2008, 2: $136-159$

5 Schnell M, Garcia-Etxarri A, Huber A J, et al. Nat Photon, 2009, 3: 287-291
6 Hillenbrand R, Taubner T, Keilmann F. Nature, 2002, 418: 159-162

7 Huber A J, Deutsch B, Novotny L, et al. Appl Phys Lett, 2008, 92: 203104

8 Greffet J J, Carminati R, Joulain K, et al. Nature, 2002, 416: 61-64

9 Rockstuhl C, Salt M G, Herzig H P. J Opt Soc Am B, 2005, 22: $481-487$

10 Schuller J A, Taubner T, Brongersma M L. Nat Photon, 2009, 3: 658

11 Renger J, Grafström S, Eng L M, et al. Phys Rev B, 2005, 71: 075410

12 Neubrech F, Pucci A, Cornelius T W, et al. Phys Rev Lett, 2008, 101: 157403

13 Xu H X, Aizpurua J, Käll M, et al. Phys Rev E, 2000, 62: 4318

14 Anderson M S. Appl Phys Lett, 2003, 83: 2964

15 Aizpurua J, Taubner T, de Abajo F J G, et al. Opt Express, 2008, 16: $1529-1545$

16 de Abajo J G, Howie A. Phys Rev B, 2002, 65: 115418

17 Prodan E, Radloff C, Halas N J, et al. Science, 2003, 302: 419-422

18 Inouye Y, Kawata S. Opt Lett, 1994, 19: 159

19 Taubner T, Korobkin D, Urzhumov Y, et al. Science, 2006, 313: 1595

20 Pendry J. Phys Rev Lett, 2000, 85: 3966

21 Fang N, Lee H, Sun C, et al. Science, 2005, 308: 534-537 